\title{
$\mathfrak{S} \mathfrak{f} \mathfrak{a} \mathfrak{t} \mathfrak{u} \mathfrak{t}$ \\ Des
}

\section{beutiden Iuxifentages.}

$\S 1$.

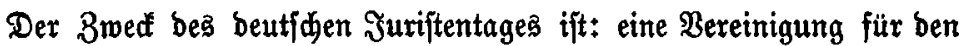
lebenbigen Meinungăaustaufd und ben perjönliden Berfehr unter ben

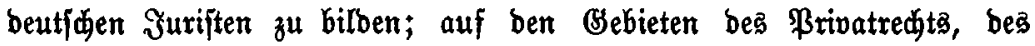
Prozeffes und beas Strafredts ben Forberungen nah einheitlidjer Ent= midélung immer größßere $\mathfrak{A n e r f e n n u n g ~ z u ~ v e r f d a f f e n , ~ b i e ~ S ̧ i n b e r n i f f e , ~}$ welde biefer Entroifelung entgegenitehen, zul bezeidnen unb fid über $\mathfrak{B o r}$

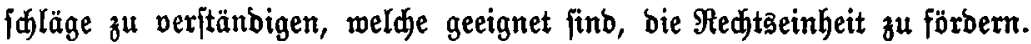

$\S 2$.

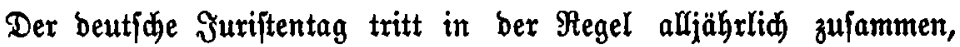
bod) ift bie ftänbige Deputation ermädtigt, aus Bränden ber 3wed"

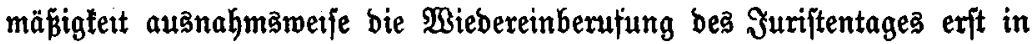
bem auf beffen leb̨ten 3 úfammentritt folgenben zmeiten Salenberjahre vorzunehmen.

\section{$\S 3$.}

3ur Mitgliebjhaft beredigt find bie beutiden Midjter, Staatsan=

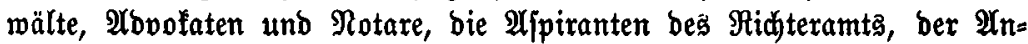

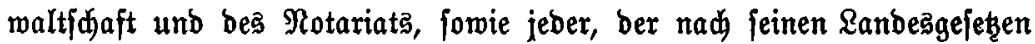

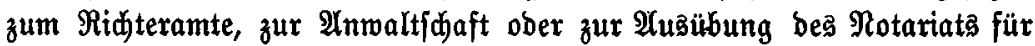

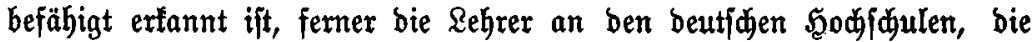
Mitglieber ber gelebrten $\mathfrak{A f a b e m i e n , ~ b i e ~ D o f t o r e n ~ b e r ~ \Re e d h t e ~ u n d ~ b i e ~}$ redisgelehrten Mitglieber ber Berwaltungăbegöroen. 
$\S 4$.

Die Mitgliebjøaft beginnt mit bem Empfange ber Mitgliebąarte. Sie beredftigt zur Teilnahme an ben Berhandlungen und an ber 2tbjtimmung.

$\S 5$.

Der Beitrag ber Befellidafts̄mitglieber beträgt Sedjs Mart jährylid

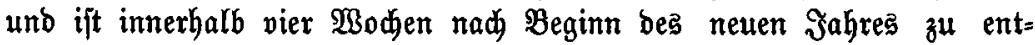

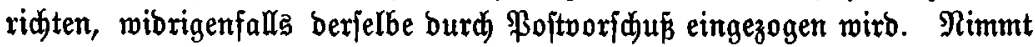

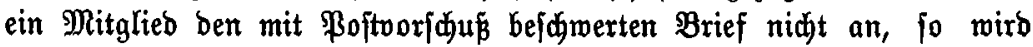

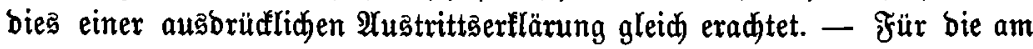

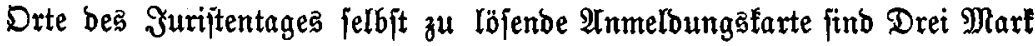
zu entriditen.

\section{$\S 6$.}

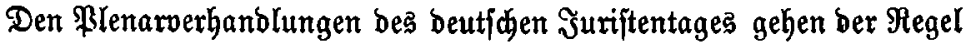
nad 2 Xthteilungaberatungen voraus. $3 \mathfrak{u}$ biefem 3 weffe werben burd frei= willige Einzeidynungen ber Mitglieber folgende vier $\mathfrak{A b t e i l u n g e n ~ g e b i l b e t : ~}$

1) AVbteilung für Brivatredt, insbejondere Dbligationen= und $\mathfrak{P f a n d}=$ redit, juriftifdes Studium und praftifde âdusbilbung.

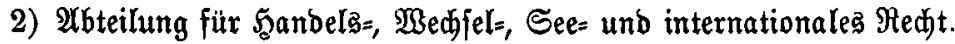

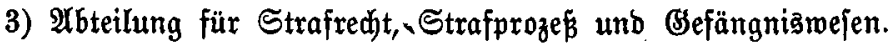

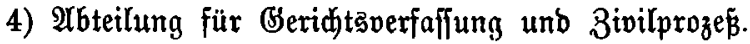

Die Arbteilungen wählen ihre Borfikenben, Sdjriftfüfrex, Beriđt= eritatter und benadridtigen ben Boritzenden ber Plenarver[ammlung (§ 7), jobald ihre Beratungen über einzelne Begenjtände gefdilofien find; ihre 2Anträge find fadriftlid) zu fafjen. Jn jeber $\mathfrak{A b t e i l u n g ~ f t i m m e n ~}$ nur Diejenigen mit, meldhe fid in bie betreffende 2rbteilung bereits ein= gezeidnet haben.

Sämtlidje Bejdlüffe ber 2Ybteilungen werben in ber \$lenarver= fammlung mitgeteilt. Es findet jebod eine Erörterung und (Entidjeibung im \$lenum nur bann ftatt, wenn biejelbe von ber betreffenden $\mathfrak{A} b$ tei= lung vorgefdlagen, ober wenn fie von minbejtens zehn Mitgliebern be= antragt und von ber \$lenarberfammlung bejhloffen wirb. ütber bie Borfrage, ob- bem von mindeftens zehn $\mathfrak{M i t g l i e b e r n}$ geftellten 2 Antrage auf \$lenarentfjeibung ftattzugeben, wiro nur einem ber ântragiteller und bem Beridbterftatter baş $\mathfrak{B o r t}$ exteilt.

\section{$\S 7$.}

Die Berhandlungen ber \$lenarverfammlung leitet ein Boritzender, meldter für bie Dauer eines jeben Juuriftentages in ber erften \$lenarvers fammlung burd Stimmzettel ober Affflamation gemählt wirb. Derfelbe 
benennt zmei biß vier Stellvertreter und vier Sdriftfübrer. Er beftimmt bie Tagežorbnung und fann einzelne Begenftänbe, ohne Borberatung in ben Abteilungen (§ 6), unmittelbar zur \$lenarberatung ftellen. 22ud ift ex befugt, Ridtmitglieber ala Zuhörer zužulaffen.

$\S 8$.

Bei allen Bejd̆lüfīen ber \$lenarverfammlung und ber Albteilungen entfdeibet einfache Majorität ber anmejenden Mitglieber, bei allen $\mathfrak{B a h l e n ~}$ relative Majorität unb im Falle ber Stimmengleidheit bas $\mathcal{L}_{0}$ s.

\section{$\S 9$.}

Wiro Sdhluk ber Debatte beantragt, fo wiro über biefen $\mathfrak{A n t r a g}$ fofort abgejtimmt. In ber ßlenarverjammlung find alle 2Anträge mit

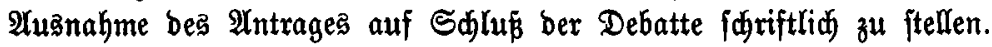

$\S 10$.

Bor bem Sălufje eines jeben Jurriftentageả wirb von ber \$lenar= verjammlung burd Afflamation ober in einem einzigen Sfrutinium burd Stimmzettel eine aus neunzehn Mitgliebern und bem Fräfíbenten beả lek̨ten Jutrijtentages als Égrenpräfibenten bejtehende ftänbige Deputa= tion gemählt. Die Rifte ber zur Affllamation vorzuj dlagenben ßerfonen wirb burd) ben Präjtibenten ber Plenarverfammlung, feine Stellvertreter unb je zehn von jeber $\mathfrak{A b t e i l u n g}$ gewählte Bertrauenämänner gemein= idaftlid feitgejtellt.

Die jtändige Deputation hat folgenbe Befugniffe und Obliegenheiten:

1) Fie forgt für bie $\mathfrak{2}$ uăführung ber von bem J̃uriftentage gefaßjten

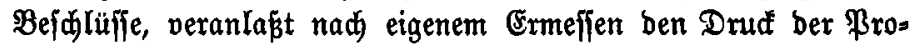
tofolle und Borlagen, bemirft bie Berteilung ber Drudfjaden an bie MRitglieber und verwahrt alle âtten und Safriftftiufe des Surijtentages;

2) fie beftimmt Beit und Drt bes nädfften Suriftentages, trifft bie für benfelben nötigen Borbereitungen, erläp̆t bie Cinlabungen, ftellt bie vorläufige Tageß̧orbnung auf, wobei fie in ber ßtegel nur bie bis zum 31. Mai bes laufenben Jahres eingegangenen

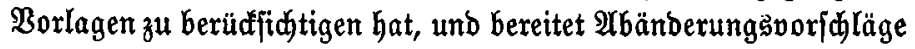
in Betreff ber Sejejäftsononung für bie \$lenarverjammlung vor;

3) Fie nimmt bie Beitrittberflärnungen neuer Mitglieber entgegen, fer= tigt bie Mitgliebsfarten auș, empfängt bie Beiträge, beftreitet bie 2 usgaben und legt ber folgenden Deputation Rechnung; 
4) Fie ergänzt fid felbit, falls eins ober mehrere Mitglieber mährend

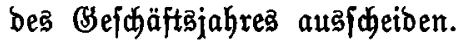

Die Deputation mählt aus ihrex Mitte einen Borfikenden, einen Sdriftführex, weldher ein von ber Deputation feitzujesenbes \$aujdquan=

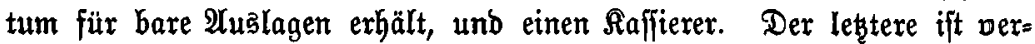
pflidłtet, Der ftändigen Deputation bei ihrem jebesmaligen Bufammentritt einen Saffenabjaluk vorzulegen. Die Deputation läßjt burd eines ober

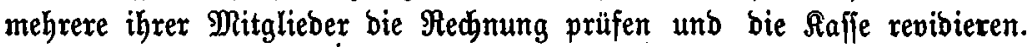

Die Deputation bejtimmt Ort und Beit ihrer Bufammenfunft. Bur

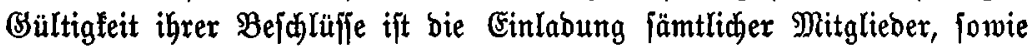
bie Mitwirfung von wenigitens fünf Mitgliebern erforberlich.

\section{$\S 11$.}

Ibänberungen bieję Statuta fönnen zmar von ber Blenarverjamm= Iung burd einfade Stimmenmehrbeit, jebod nur auf fodriftliden 2 Intrag, Der vier Moden vor bem 3 uffammentritt beş Jüriftentages ber ftänbigent Deputation (\$ 10) überreidft morben, befolloffen werben. 
$(\mathfrak{b} \mathfrak{a} \mathfrak{t} \mathfrak{a} \mathfrak{d} \mathfrak{t} \mathfrak{e} \mathfrak{n}$.

(Erjter Bando) 
